\title{
A new rapid screening program based on risk scores for COVID-19 patients
}

\author{
Ru Chen ${ }^{1} \cdot$ Guilan $\mathrm{Xu}^{2} \cdot$ Lihui Yang ${ }^{3} \cdot$ Zelin Deng ${ }^{4} \cdot$ Qing $\mathrm{Hu}^{1} \cdot \mathrm{Hao} \mathrm{Hu}^{1} \cdot$ Zhen Wang $^{1,2}$ (I)
}

Received: 17 April 2020 / Accepted: 10 October 2020 / Published online: 27 October 2020

(c) Società Italiana di Medicina Interna (SIMI) 2020

\begin{abstract}
We aimed at establishing a new COVID-19 risk scores, serving as a guide for rapidly screening the COVID-19 patients in order to reduce the risk of COVID-19 hospital-related transmission. As the COVID-19 disease is breaking out across the world, hospital-related transmission is one of the main factors accountable for the spread of COVID-19. For COVID-19 prevention it is urgent to establish a fast and efficient screening strategy for the COVID-19 patients. We analyzed 335 patients (including 124 patients with COVID-19). Five significant clinical attributes were selected as the components for establishing a COVID-19 risk score system, and every attribute was assigned a specific score according to their respective odds ratio values. We also compared three different screening schemes (Scheme I: temperature higher than $37.2^{\circ} \mathrm{C}$ on admission, Scheme II: exposure to a source of transmission within 14 days in addition to fever, Scheme III: our new COVID-19 risk score) in terms of their respective receiver operating characteristic (ROC) curves, so as to evaluate their respective screening effectiveness. Five significant risk factors, which were exposed to a source of transmission (9 points), cluster onset (6 points), history of fever or temperature higher than $37.2{ }^{\circ} \mathrm{C}$ on admission (4 points), cough (1 point) and other atypical symptoms (1 point), were ultimately selected from many candidates to construct the new rapid COVID-19 screening program. Based on the screening scheme, the patients were quickly divided into three subgroups according to their respective COVID-19 risk scores: low risk ( $\leq 6$ points, risk $<10 \%)$, medium risk ( $7-13$ points) and high risk ( $\geq 14$ points, risk $>80 \%$ ). When the score of 10 points was selected as a cut-off point for differentiating the patients with COVID-19 from all of the other patients, the sensitivity was $93.6 \%$, with a specificity of $86.3 \%$. The area under the ROC curve (AUC) of COVID-19 risk score system was $0.96(P=0.000)$, much higher than the AUCs of Scheme I $(0.56, P=0.000)$ and Scheme II $(0.85, P=0.000)$, respectively. Our COVID-19 risk score system can help the clinicians effectively and rapidly identify and differentiate the patients with COVID-19 infections, to be mainly used in those areas where COVID-19 still exhibits epidemiological characteristics.
\end{abstract}

Keywords COVID-19 $\cdot$ Risk scores $\cdot$ Screening program

Electronic supplementary material The online version of this article (https://doi.org/10.1007/s11739-020-02534-6) contains supplementary material, which is available to authorized users.

Zhen Wang

wz_doc@126.com

1 Neurological Department, The Third Xiangya Hospital of Central South University, Changsha, Hunan, China

2 Neurological Department, Changsha Central Hospital, No. 161, Shaoshan South Road, Changsha, Hunan, China

3 Infectious Disease Department, The First Hospital of Changsha City, Changsha, Hunan, China

4 School of Computer and Communication Engneering, Changsha University of Science and Technology, Changsha, Hunan, China

\section{Introduction}

In early December 2019, some pneumonia cases of unknown origins were identified in Wuhan City, Hubei Province, China [1]. High-throughput sequencing has revealed a novel beta coronavirus, currently named 2019 novel coronavirus, as their cause [2]. The coronavirus disease 2019 (COVID-19) continued to spread to the world in the following 3 months. The World Health Organization recently declared the COVID-19 a public health emergency of international concern [3].

Hospital-related transmission is one of the main reasons for the spread of COVID-19. In a single-center case of 138 hospitalized patients with COVID-19 in Wuhan, China, hospital-related transmission was presumably accountable for 
$41 \%$ of the inpatient infections [4]. The emergency department is a high-risk area of cross infection as many patients are concentrated there. So it is very important to screen and separate the patients with COVID-19 quickly in order to reduce the risk of hospital-related transmission. The management of patients with COVID-19 is a new challenge for emergency medicine [5].

Here, we have collected the clinical data of both the COVID-19 and non-COVID-19 patients, who visited the emergency department in the same period in Changsha City, Hunan Province, a neighbor to the epicentral Hubei Province. We aim to establish a rapid COVID-19 risk scoring scheme, serving to guide the efficient screening and separation of the COVID-19 patients.

\section{Method}

\section{Patients}

Patients with COVID-19: We retrospectively retrieved the clinical data of COVID-19 patients hospitalized in North District of Changsha First Hospital (Changsha, Hunan Province) on February 8th, 2020. Cases were diagnosed according to the WHO interim guidance [6] and confirmed with 2019-nCoV real-time reverse-transcriptase polymerasechain-reaction (RT-PCR) assays for nasal and pharyngeal swab specimens.

Patients without COVID-19 (control group): We retrospectively retrieved the clinical data of patients in the emergency department in The Third Xiangya Hospital of Central South University, Changsha from February 5th to 7th, 2020. After 14 days of observation, COVID-19 was excluded for all of these patients (i.e. none of the control group patients was on the list of patients confirmed with COVID-19).

This study was carried out in February 2020 during the Chinese New Year and amidst the peak of the COVID-19 epidemic in China. The hospital's Research Ethics Committee was on leave according to the government regulations, but we had to carry out the study soon as possible, hence our being unable to get a research ethics clearance before starting data collection. However, we had done our best to protect the rights and interests of our research subjects.

Information of patients was anonymized and desensitized before analysis, and we made sure that there was no disclosure of sensitive information, such as the names and addresses of the COVID-19 patients, throughout the study.

\section{Clinical assessment}

The patients' demographic attributes, epidemiological characteristics, medical histories, clinical symptoms and computed tomographies were extracted from the hospitals' electronic medical records. Exposure to a source of transmission within 14 days before symptom onset referred to one of the three situations taking place within two weeks: a travel history from Wuhan City; in contact with patients with fever or respiratory symptoms from Wuhan; epidemiologically connected to COVID-19 infections. Clustered onsets were identified as one or more of the close contacts developed a disease simultaneously.

\section{Statistical analysis}

First, continuous variables were recorded as either means and standard deviations or medians and interquartile ranges (IQR) as appropriate. Categorical variables were summarized as number counts and percentages in each statistical category. Wilcoxon rank-sum tests were applied to the continuous variables, while chi-square tests and Fisher's exact tests were used for categorical variables as appropriate. Second, the potential risk factors were analyzed using logistic regression. According to the odds ratios (ORs) and the clinical situations, COVID-19 risk scores were established. Third, three different screening programs were compared: Scheme I, temperature higher than $37.2{ }^{\circ} \mathrm{C}$ on admission; Scheme II, exposure to a source of transmission within 14 days in addition to fever; and Scheme III, COVID-19 risk scores. We computed the receiver operating characteristic (ROC) curves of the above three screening programs, respectively, and the areas under the receiver operator curves (AUCs) were then compared to assess their clinical performance.

\section{Results}

\section{Demographic and clinical characteristics}

Patients with COVID-19: As of February 8th, 2020, 196 COVID-19 patients were confirmed in Changsha [7], and we have collected 124 of the confirmed cases. For all of the patients, 2019-nCoV RT-PCR assays for nasal and pharyngeal swab specimens were tested positive. Every one of the patients underwent chest computer tomography on admission, and $96.3 \%$ manifested pneumonia. Six patients, who had no abnormal radiological results, were diagnosed by symptoms in addition to positive RT-PCR findings. One patient, who had no symptom and a normal radiological result, was diagnosed by history of epidemiological exposure together with a positive RT-PCR result.

The demographic and clinical characteristics are shown in Table 1. The median age was 42.5 years (IQR, 34.3-54 years old) and $50.8 \%$ of the patients were females. In our study, the COVID-19 disease was diagnosed throughout the whole spectrum of age, with the youngest patient being 1 year old 
Table 1 Clinical characteristics of the patients in 2019-nCoV ARD group and control group

\begin{tabular}{|c|c|c|c|c|}
\hline Clinical characteristics & All patients $(n=335)$ & COVID-19 group $(n=124)$ & Control group $(n=211)$ & $P$ \\
\hline Age, median (IQR), years & $42(32-57)$ & $42.5(34.3-54)$ & $42.0(31-59)$ & 0.99 \\
\hline Female sex-no., $\%$ & $156(50.8 \%)$ & $63(50.8 \%)$ & $93(44.1 \%)$ & 0.26 \\
\hline Exposure to source of transmission within 14 days ${ }^{\mathrm{a}}-$ no., $\%$ & $162(48.4 \%)$ & $117(94.4 \%)$ & $45(21.3 \%)$ & 0.00 \\
\hline Cluster onset $^{\mathrm{b}}$ & $49(14.6 \%)$ & $41(33.1 \%)$ & $8(3.8 \%)$ & 0.00 \\
\hline \multicolumn{5}{|l|}{ Symptoms-no., \% } \\
\hline History of fever & $156(46.6 \%)$ & $101(81.5 \%)$ & $55(26.1 \%)$ & 0.00 \\
\hline Temperature on admission, average $(95 \% \mathrm{CI}),{ }^{\circ} \mathrm{C}$ & $36.7(36.3-37.4)$ & $37.0(36.5-37.5)$ & $36.6(36.2-37.2)$ & 0.00 \\
\hline Temperature on admission $>37.2^{\circ} \mathrm{C}$ & $94(28.1 \%)$ & $44(35.5 \%)$ & $50(23.7 \%)$ & 0.00 \\
\hline Scoring establishment & $94(28.1 \%)$ & $44(35.5 \%)$ & $50(23.7 \%)$ & 0.02 \\
\hline Cough & $150(44.8 \%)$ & $80(64.5 \%)$ & $70(33.2 \%)$ & 0.00 \\
\hline Sore throat & $43(12.8 \%)$ & $19(15.3 \%)$ & $24(11.4 \%)$ & 0.31 \\
\hline Nasal congestion and rhinorrhea & $18(5.4 \%)$ & $5(4 \%)$ & $13(6.2 \%)$ & 0.46 \\
\hline Chest congestion & $43(12.8 \%)$ & $10(8.1 \%)$ & $33(15.6 \%)$ & 0.06 \\
\hline Shortness of breath & $34(10.1 \%)$ & $9(7.3 \%)$ & $25(11.8 \%)$ & 0.20 \\
\hline Anorexia & $14(4.2 \%)$ & $12(9.7 \%)$ & $2(0.9 \%)$ & 0.00 \\
\hline Nausea or vomiting & $30(9 \%)$ & $7(5.6 \%)$ & $23(10.9 \%)$ & 0.12 \\
\hline Diarrhea & $21(6.3 \%)$ & $6(4.8 \%)$ & $15(7.1 \%)$ & 0.49 \\
\hline Chill & $18(5.4 \%)$ & $7(5.6 \%)$ & $11(5.2 \%)$ & 1.00 \\
\hline Fatigue & $50(14.9 \%)$ & $31(25 \%)$ & $19(9 \%)$ & 0.00 \\
\hline Myalgia or arthralgia & $15(4.5 \%)$ & $13(10.5 \%)$ & $2(0.9 \%)$ & 0.00 \\
\hline Vertigo & $20(6 \%)$ & $6(4.8 \%)$ & $14(6.6 \%)$ & 0.64 \\
\hline Headache & $28(8.4 \%)$ & $20(16.1 \%)$ & $8(3.8 \%)$ & 0.00 \\
\hline Belching & $1(0.2 \%)$ & $1(0.8 \%)$ & $0(0 \%)$ & 0.37 \\
\hline Coexisting disorders-no., $\%$ & $71(21.2 \%)$ & $25(20.2 \%)$ & $46(21.8 \%)$ & 1.00 \\
\hline Chronic obstructive pulmonary disease & $6(1.8 \%)$ & $0(0.0 \%)$ & $6(2.8 \%)$ & \\
\hline Diabetes & $22(6.6 \%)$ & $8(6.5 \%)$ & $14(6.6 \%)$ & \\
\hline Hypertension & $37(11.0 \%)$ & $19(15.3 \%)$ & $18(8.5 \%)$ & \\
\hline Coronary heart disease & $15(4.5 \%)$ & $3(2.4 \%)$ & $12(5.7 \%)$ & \\
\hline Cerebrovascular diseases & $7(2.1 \%)$ & $4(3.2 \%)$ & $3(1.4 \%)$ & \\
\hline Cancer & $8(2.4 \%)$ & $0(0.0 \%)$ & $8(3.8 \%)$ & \\
\hline Hepatitis B infection & $6(1.8 \%)$ & $1(0.8 \%)$ & $5(2.4 \%)$ & \\
\hline Chronic renal diseases & $5(1.5 \%)$ & $1(0.8 \%)$ & $4(1.9 \%)$ & \\
\hline Immunodeficiency & $2(0.6 \%)$ & $1(0.8 \%)$ & $1(0.5 \%)$ & \\
\hline
\end{tabular}

Wilcoxon rank-sum tests were applied to continuous variables, chi-square tests and Fisher's exact tests were used for categorical variables $I Q R$ interquartile range

${ }^{a}$ Exposure to a source of transmission within 14 days includes a travel history from Wuhan City, in contact with the patients with fever or respiratory symptoms from Wuhan, or epidemiologically connected to COVID-19 infections

${ }^{\mathrm{b}}$ Cluster onset: one or more of the close contacts developed a symptom simultaneously

and the oldest 84 . A history of epidemiological exposure was documented in $94.4 \%$ of the population and $33.1 \%$ of them had cluster onset records. Five patients (2.3\%) did not have any clear history of epidemiological exposure.

The clinical manifestations of the patients are various. Fifteen types of clinical manifestations were collected here. Fever (81.5\%) and cough (64.5\%) were the commonest symptoms. Next by decreasing order were fatigue (25\%), headache $(16.1 \%)$, sore throat $(15.3 \%)$, myalgia or arthralgia (10.5\%), anorexia (9.7\%), chest congestion $(8.1 \%)$, shortness of breath (7.3\%), chill(5.6\%), nausea or vomiting (5.6\%), diarrhea (4.8\%), vertigo (4.8\%), nasal congestion $(4.0 \%)$ and belching $(0.8 \%)$. Four $(3.2 \%)$ of the 124 patients had no clinical symptom. The median temperature on admission was $37.0{ }^{\circ} \mathrm{C}$ (IQR, $\left.36.5-37.5\right)$ and $35.5 \%$ of the patients had temperatures higher than $37.2^{\circ} \mathrm{C}$. $20.2 \%$ of patients had at least one underlying disorder (i.e. hypertension, chronic obstructive pulmonary disease, etc.).

Patients without COVID-19 (control group): There were 255 patients in the emergency ward of internal medicine of 
The Third Xiangya Hospital of Central South University between February 5th and 7th. The data of 211 of these patients were collected. Another 43 of the patients were excluded due to incomplete electronic medical records, and one other patient was excluded because of unclear diagnosis. This last patient had an onset of fever and quickly developed into respiratory failure. The patient's chest CT showed viral pneumonia, but they had no history of exposure to any source of transmission, and three 2019-nCoV RT-PCR assays were tested negative. Because the diagnosis was not clear, it was not included in our data analysis.

Out of the 211 patients in the control group, 53 with contact histories or exposure to cluster onsets were given RTPCR assays for at least twice, and all of their results were negative. For the rest 158 patients in the group, only 23 of them were able to receive an RT-PCR test once, and their results were all negative, too.

Their demographic and clinical characteristics are shown in Table 1. The control group's median age was 42.0 years old (IQR, 31-59 years old) and $44.1 \%$ were females. A history of epidemiological exposure was documented in 45 (21.3\%) of the patients and cluster onset records were documented in eight (3.8\%). For these 53 patients, however, the chest CTs were normal and RT-PCR results were negative.

For the control group, we also collected 14 types of clinical manifestations, and their frequencies were as follows: fever $(26.1 \%)$, cough $(33.2 \%)$, fatigue $(9 \%)$, headache $(3.8 \%)$, sore throat $(11.8 \%)$, myalgia or arthralgia $(0.9 \%)$, anorexia $(0.9 \%)$, chest congestion(15.6\%), shortness of breath $(7.3 \%)$, chill $(5.2 \%)$, nausea or vomiting (10.9\%), diarrhea (7.1\%), vertigo (6.6\%) and nasal congestion (6.2\%). $21.8 \%$ of the patients had at least one underlying disorder.

After comparing the two groups (COVID-19 group versus control group), six of the manifestations, i.e. fever, cough, anorexia, fatigue, myalgia or arthralgia and headache, were shown to be more common in the COVID-19 group than the other (their P values were all smaller than 0.001). Meanwhile, there was no statistically significant difference in the distribution of other clinical symptoms (including temperature on admission) or co-morbid diseases between the two groups.

\section{Establishment of scoring scheme}

The variables were assigned while modeling. To facilitate translation into a clinical rating scale, this part of the data was processed as shown in Table 2. As far as the grouping of patients was concerned, the control group was represented by 0 and the COVID-19 group by 1 . For each of the five risk factors, i.e. (1) exposure to a source of transmission within 14 days, (2) cluster onset, (3) history of fever or temperature on admission higher than $37.2^{\circ} \mathrm{C}$, (4) cough and (5) other atypical symptoms, a yes was represented by 1 , and
Table 2 Assignment of variables

\begin{tabular}{|c|c|}
\hline Variables & Assignment method \\
\hline Group & $\begin{array}{l}\text { Control group =0; } \\
\text { COVID-19 } \\
\text { group }=1\end{array}$ \\
\hline $\begin{array}{l}\text { Exposure to source of transmission within } \\
14 \text { days-no., } \%\end{array}$ & $\mathrm{No}=0 ;$ yes $=1$ \\
\hline Cluster onset & No $=0 ;$ yes $=1$ \\
\hline $\begin{array}{l}\text { History of fever or temperature on admission } \\
\text { higher than } 37.2^{\circ} \mathrm{C}\end{array}$ & $\mathrm{No}=0 ;$ yes $=1$ \\
\hline Cough & No $=0 ;$ yes $=1$ \\
\hline Other atypical symptoms & No $=0 ;$ yes $=1$ \\
\hline
\end{tabular}

Other atypical symptoms: included such symptoms as sore throat, nasal congestion, chest congestion, shortness of breath, anorexia, nausea or vomiting, diarrhea, chill, fatigue, myalgia or arthralgia, vertigo, headache and belching

a no was represented by 0 . The final risk factor of other atypical symptoms included such symptoms as sore throat, nasal congestion, chest congestion, shortness of breath, anorexia, nausea or vomiting, diarrhea, chill, fatigue, myalgia or arthralgia, vertigo, headache and belching.

These five candidate variables were all statistically significant in the logistic regression analysis and formed our final set of predictors, and the ORs of the five factors are shown in Table 3. The OR of the exposure to a source of transmission was the highest (123.9); next by decreasing order were the ORs of cluster onset (30.2), history of fever or temperature higher on admission (12.6), cough (3.8) and other atypical symptoms (3.8). When assigning scores to each of the risk factors, we have taken both the OR values and the clinical situations into account. So the maximum score for a risk factor was set at nine points, while the minimum was set at one point. That is, the higher the OR, the higher the risk factor's score. The scores of the five screening factors are shown in Table 3.

We computed a total score for each of the patients by summing up all of the patient's 5-factor scores. In our patient group, the highest total score was 21 , whereas the lowest was 0 . The subtotal of the scores indicated the likelihood of an incidence of COVID-19, as shown in Fig. 1. As the figure exhibited, the higher the score, the greater the possibility of COVID-19 was for the patient. Patients scoring 3 or 18 points did not exist, so these two sets of data were not available.

The critical points on the graph occurred at 6 and 14 points, respectively. Consequently, we divided all of the patients into three subgroups. Group 1 (low risk) patients had their scores ranging from 0 to 6 , with the potential COVID-19 risk lower than 10\%. Group 2 (medium risk) had their scores ranging from 7 to 13 , with the potential COVID-19 risk between 10 and 80\%. Group 3 (high risk) 
had their scores ranging from 14 to 21 , and the risk for the patients acquiring COVID-19 was higher than $80 \%$.

We drew the ROC curves (Fig. 2) for the COVID-19 risk scores and calculated the sensitivity, specificity and Yuen index according to the ROCs. The cut-off point was 10 points, which had the largest Yuen index of 0.8 .

We also computed the ROC values (Fig. 2) for each of the three screening programs we had tested in our study, in an attempt to evaluate which one of them had the highest accuracy in distinguishing COVID-19 patients from nonCOVID-19 patients. These schemes were as follows: Scheme
I, temperature higher than 37.2 on admission; Scheme II, exposure to source of transmission within 14 days and fever; and Scheme III, COVID-19 risk scores (our own).

As Table 4 shows, the AUC value of our proprietary COVID-19 risk score program was 0.96 (0.94-0.98), whereas the AUCs of Scheme I and II were 0.56 $(0.50-0.63)$ and $0.85(0.80-0.90)$, respectively. When ten points was used as the cut-off for the COVID-19 screening (i.e. only patients with a score greater than or equal to 10 points were assigned to the COVID-19 group), only eight COVID-19 patients and 29 non-COVID-19 patients were
Table 3 Logical regression analysis result: the association of risk factors with COVD-19 disease

Fig. 1 The predictability of COVID-19 scores

Fig. 2 ROC curves of three schemes. Scheme I: temperature higher than 37.2 on admission, Scheme II: exposure to a source of transmission within 14 days in addition to fever, Scheme III: COVID-19 risk scores

\begin{tabular}{lllll}
\hline & $\begin{array}{l}\text { Regression coefficient } \\
(M \pm S D)\end{array}$ & Odds ratio (95\% CI) & $P$ & Scores \\
\hline $\begin{array}{l}\text { Exposure to source of transmis- } \\
\text { sion within 14 days-no., } \%\end{array}$ & $4.8 \pm 0.6$ & $123.9(36.6-419.0)$ & 0.00 & 9 \\
$\begin{array}{l}\text { Cluster onset } \\
\text { History of fever or tempera- } \\
\text { ture higher than } 37.2^{\circ} \mathrm{C} \text { on }\end{array}$ & $3.4 \pm 0.7$ & $30.2(7.1-129.4)$ & 0.00 & 6 \\
admission & $2.5 \pm 0.4$ & $12.6(5.2-30.4)$ & 0.00 & 4 \\
Cough & $1.3 \pm 04$ & & & \\
Other atypical symptoms & $1.3 \pm 0.4$ & $3.8(1.6-8.9)$ & 0.00 & 1 \\
\hline
\end{tabular}

Other atypical symptoms: sore throat, nasal congestion, chest congestion, shortness of breath, anorexia, nausea or vomiting, diarrhea, chill, fatigue, myalgia or arthralgia, vertigo, headache and belching
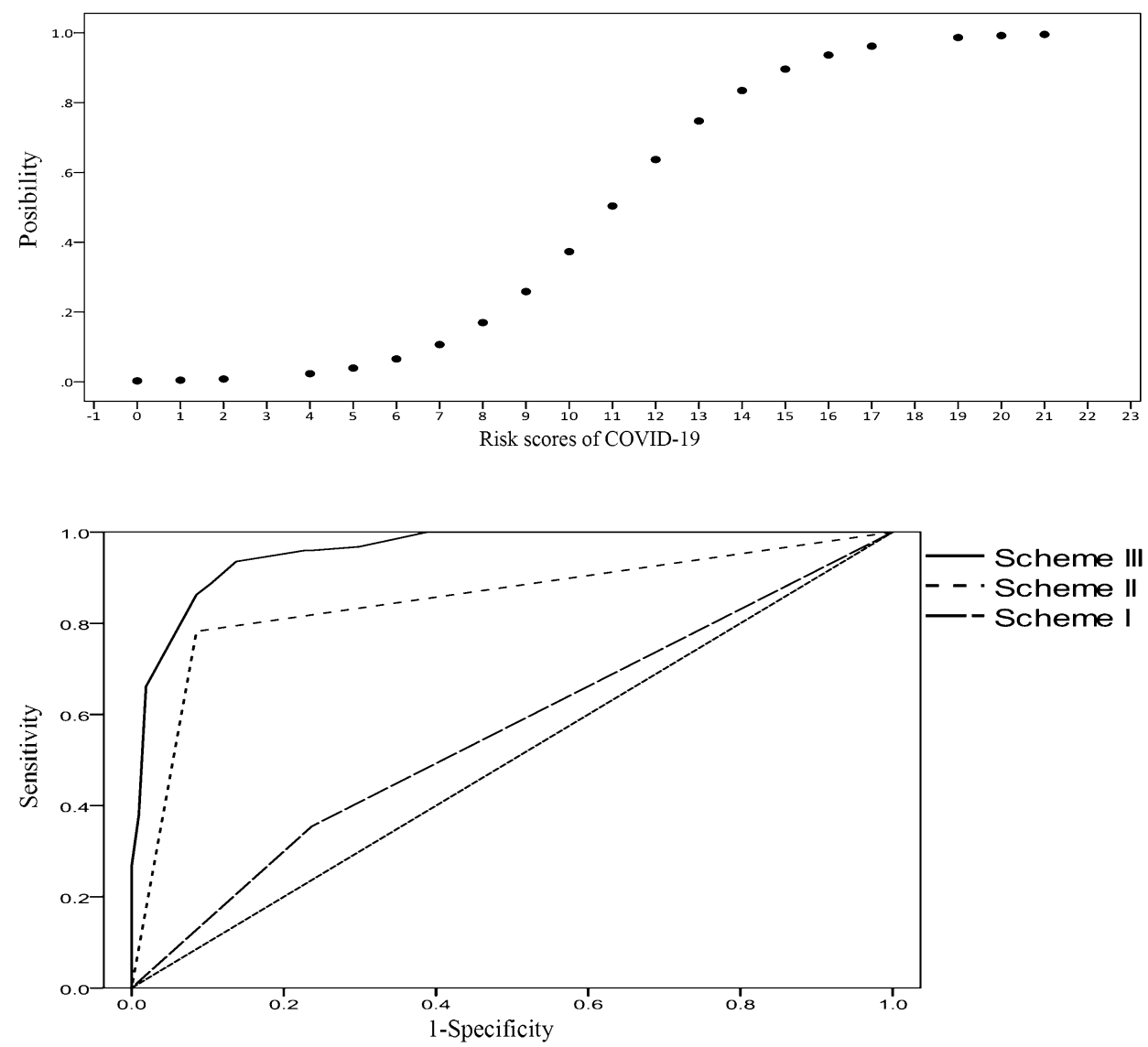
Table 4 The classification results of three schemes

\begin{tabular}{llll}
\hline \multicolumn{1}{c}{ Scheme I } & Scheme II & Scheme III \\
\hline COVID-19 group & & \\
Correct & 44 & 97 & 116 \\
Error & 80 & 27 & 8 \\
Control group & & & \\
Correct & 161 & 193 & 182 \\
Error & 50 & 18 & 29 \\
AUC (95\% & $0.56(0.50-$ & $0.85(0.80-$ & $0.96(0.94-0.98)$ \\
CI) & $0.63)$ & $0.90)$ & \\
Sensitivity & $35.5 \%$ & $78.20 \%$ & $93.50 \%$ \\
Specificity & $76.3 \%$ & $91.50 \%$ & $86.30 \%$ \\
Accuracy & $61.2 \%$ & $86.60 \%$ & $89 \%$ \\
\hline
\end{tabular}

Scheme I: temperature higher than $37.2{ }^{\circ} \mathrm{C}$ on admission; Scheme II, exposure to a source of transmission within 14 days plus fever; Scheme III, COVID-19 risk scores (the patients with a score greater than or equal to 10 points were assigned to the COVID-19 group)

misclassified (the test's sensitivity was $93.6 \%$, with a specificity of $86.3 \%$ ). In comparison, Scheme I misclassified 80 COVID-19 patients and 50 non-COVID-19 patients (with a sensitivity of $35.5 \%$ and a specificity of $76.3 \%$ ). Scheme II had 27 COVID-19 patients and 18 non-COVID-19 patients misclassified (with a sensitivity of $78.2 \%$ and a specificity of $91.5 \%$ ). We thus concluded that the COVID19 risk score scheme performed the best among the three.

\section{Validation}

Based on the same criteria, we have collected again the data of patients, who visited the emergency and fever clinic departments in Xiangya No. 3 Hospital on February 9 . A total of 96 patients were collected, including six COVID-19 cases and 90 non-COVID-19 cases. The information of the patients is shown in Supplementary Appendix. Each patient has been evaluated according to the COVID-19 risk score. The scores of six COVID-19 patients were, respectively, 20,16, 15, 15, 15 and 9 .

According to the trichotomy (low-risk group $\leq 6$, medium-risk group $=7-13$, high-risk group $\geq 14$ ), there were no COVID-19 patients in the low-risk group, one $(16.7 \%)$ in the medium-risk group, and five $(83.3 \%)$ in the high-risk group. In comparison, there were $79(87.7 \%)$ non-COVID-19 patients in low-risk, nine (10\%) in medium-risk, and two (2.2\%) in high-risk. Alternatively, according to the dichotomy (low-risk group $\leq 9$, highrisk group $\geq 10)$, one (16.7\%) COVID-19 patient was in the low-risk group and five (83.3\%) were in high-risk. In comparison, 79 (87.7\%) non-COVID-19 patients were in low-risk and $11(12.2 \%)$ in high-risk.

\section{Discussion}

Given that the COVID-19 disease is continuously breaking out around the world, we have aimed at establishing a risk score system that can make a rapid screening of COVID19 patients without imaging or experimental examination. In this way, we hope that it can help significantly reduce the hospital-related transmission and control the disease.

In the literature, COVID-19 was reported throughout all age stages, but the majority was the young and middleaged adults. This was not to say that the elderly and children were not susceptible. A possible explanation for this pattern could be that the young and middle-aged patients were more socially active than the elderly and children. There was no difference between the sexes. Nearly $20.2 \%$ of the patients had at least one underlying disease. An epidemiological history of exposure was a significant feature of COVID-19 patients. The clinical manifestations of the patients with COVID-19 were various and lack of specificity. There were 20 clinical symptoms documented in the literature, involving the respiratory system (cough, sore throat, nasal congestion, shortness of breath, etc.), digestive system (nausea, vomiting, diarrhea, etc.), neuromuscular system (myalgia, arthralgia, vertigo, headache) and systemic infection (fever, chill, fatigue, anorexia, muscle pain, etc.). The most common symptoms, however, were fever (43\%-97.6\%) and cough (67.8\%).

In this study, the data of 124 patients with COVID19 were collected and all of them were hospitalized in Changsha, China. Changsha is a city with a population of more than seven million, with a distance of $350 \mathrm{~km}$ from Wuhan, the epicenter. The city is a typical case of imported COVID-19. Compared with the previous reports, the patients in this study were younger, but the majority was still young and middle-aged adults. There was no difference between the sexes. $20.2 \%$ of the patients had at least one underlying disorder. An epidemiological history of exposure $(94.4 \%)$ and cluster onset $(33.1 \%)$ were also significant features in our population of COVID-19 patients. Five patients did not have any clear epidemiological history or cluster onset. There were 15 documented clinical manifestations. Sputum and hemoptysis were included in cough. Conjunctivitis, confusion and abdominal pain were not documented. Fever $(81.5 \%)$ and cough (64.5\%) were also the commonest symptoms. This study was a single-center study and the patient data were limited, but still representative.

Compared with the other patients in the same area at the same time, epidemiological history and cluster onset were significant characteristics of the COVID-19 patients. The incidence of systemic infection (fever, chills, fatigue, anorexia, muscle pain), cough and headache in the patients 
with COVID-19 was higher. There was no significant difference in other symptoms between the COVID-19 patients and control group.

According to the literature, there was no significant difference in symptoms between COVID-19 and seasonal influenza, another common respiratory disease. The age of onset of influenza was 23.4 (mean), 53.8\% of the infected were males and the total disease burden was 7.2-61\%; fever was a symptom for $36-100 \%$ of the infected population, cough $40-80 \%$, shortness of breath $7-100 \%$ and diarrhea $4-25 \%$ [8-11]. Overall, the clinical manifestations of patients with COVID-19 lacked specificity, which has made it difficult to quickly identify and separate the COVID-19 patients.

Currently, single temperature measurement and epidemiological investigation were common triage strategies in use for screening patients with COVID-19. Fever was a common symptom in patients with COVID-19, and it was believed that fever existed in 78-99\% of patients [4, 11-13]. However, other studies suggested that fever was not obvious in an early stage of infection, with only $43.1 \%$ of the patients having had elevated body temperature on admission [14]. The same problem was also found in this study. Only 35.5\% of the patients had elevated body temperature on admission, and a further investigation showed that $81.5 \%$ of the patients had a recent history of fever. Therefore, it was inferred that fever was still the main feature of patients with COVID-19, but due to the thermal type, some patients' body temperatures fluctuated and were not in a state of continuous fever. Therefore, single thermometry was obviously not a sufficient measure and repeated thermometry was critical for the differentiation of patients with COVID-19.

The purpose of this study was to establish a rapid screening standard for the patients with COVID-19. The control group data were obtained from the emergency room patients in the same region during the same period. Considering the convenience of data analysis, we devised that the numbers of patients in the two groups should be similar, so the control group only included three days' data. There were five risk factors in this study: (1) exposure to a source of transmission within 14 days (9 points), (2) cluster onset (6 points), (3) history of fever or temperature on admission higher than $37.2{ }^{\circ} \mathrm{C}$ (4 points), (4) cough (1 point) and (5) other atypical symptoms (1 point). Atypical symptoms included 13 such symptoms as sore throat, nasal congestion or rhinorrhea, chest congestion, shortness of breath, anorexia, nausea or vomiting, diarrhea, chill, fatigue, myalgia or arthralgia, vertigo, headache and belching. In this study, we did not collect the data of patients with abdominal pain, confusion and conjunctival congestion [4, 13-15], but by reviewing the literature, we have decided that the three symptoms could also be included as atypical symptoms.

According to their respective COVID-19 risk scores, the patients were divided into three subgroups: low risk
(0-6 points), medium risk (7-13 points) and high risk (14-21 points). The risk of patients with COVID-19 was less than $10 \%$ in the low-risk group, but more than $80 \%$ in the high-risk group. For example, if a patient only had fever and cough, the risk score would be 5 and categorized as low-risk. If a patient had a history of exposure and diarrhea, which was an atypical symptom, at the same time, then the risk score would be 10 and thus put into the medium-risk group. If a patient had fever, cough and a history of exposure all together, then the score would be14, high risk group. If a patient without any symptom had a history of exposure and a cluster onset through close contact, then the score would be 15 , also high risk.

We also compared the performance of three different screening programs: Scheme I, temperature higher than $37.2{ }^{\circ} \mathrm{C}$ on admission; Scheme II, exposure to a source of transmission within 14 days plus fever; and Scheme III, COVID-19 risk scores. The ROC curves (Fig. 2) indicated that the COVID-19 risk score system (AUC of 0.96) had better performance than Schemes I and II (AUCs of 0.56 and 0.85 , respectively) did.

There was a risk of misclassification in any screening program, and we hoped to reduce the number of patients with misclassification as much as possible, especially those with COVID-19. When the score of 10 was recommended as the cut-off point for the COVID-19 screening, that is, only the patients with a score greater than or equal to 10 points were assigned to the COVID-19 group, 37 patients were misclassified,but only eight were COVID-19 patients. This result was comparatively better than those of Scheme I (130 patients were misclassified, where 80 were COVID-19 s) and scheme II (45 patients were misclassified, where 27 were COVID-19 s).

However, our study still has three important limitations. First, many of the risk score points were assigned according to epidemiological criteria. For the patients without any epidemiological history or cluster-onset exposure record, the system's differentiation effect is limited and thus may not be applicable. So we do not recommend direct application of the risk score system to the areas which has already had a broad diffusion of COVID-19 or where the disease has lost significant epidemiological characteristics. Second, since some of the patients in control group could not be tested by RT-PCR at the time of our data collection, despite our additional measures and checks, it is still possible that some of the patients with COVID-19 in our data set were misrepresented as 'controls.' Third, this is a single-center study and the number of patients is limited. Although the COVID-19 patients in the study account for 51 percent (124/242) of the total confirmed COVID-19 cases in our city, we still hope that the model can be further verified and validated externally in other multiple centers. We also hope that it will help more 
healthcare comrades to fight against our common enemy, the COVID-19, in a more effective and safer manner.

\section{Conclusion}

The preliminary results suggest that the COVID-19 score scheme that we have designed can be used for the rapid screening of COVID-19 patients in hospitals, and it has better performance than the current commonly used screening programs, i.e. temperature higher than $37.2^{\circ} \mathrm{C}$ on admission and exposure to a source of transmission within 14 days plus fever. Thus, we also recommend triage based on the scheme, where patients with a risk score of greater than or equal to 10 points should be quickly diverted.

Author contributions All authors had access to the data and a role in writing this manuscript.

Funding This work was supported by Youth Fund Project of Hunan Provincial Department of Education (16B006), China. Funders were involved in the analysis of data.

\section{Compliance with ethical standards}

Conflict of interest RC, GX, LY, QH, HH and ZW, none. ZD has received Youth Fund Project of Hunan Provincial Department of Education (16B006), China.

Statement of human and animal rights The research complied with the Declaration of Helsinki.

Informed consent Information of patients was anonymized and deidentified before analysis.

\section{References}

1. Huang C, Wang Y, Li X et al (2020) Clinical features of patients with 2019 novel coronavirus in Wuhan, China. Lancet. https://doi. org/10.1016/S0140-6736(20)30183-5

2. Lu R, Zhao X, Li J et al (2020) Genomic characterization and epidemiology of 2019 novel coronavirus: implications of virus origins and receptor binding. Lancet. https://doi.org/10.1016/ S0140-6736(20)30251-8

3. WHO main website. https://www.who.int. Accessed 5 Feb 2020

4. Wang D, Hu B, Hu C et al (2020) Clinical characteristics of 138 hospitalized patients with 2019 novel coronavirus-infected pneumonia in Wuhan, China. JAMA. https://doi.org/10.1001/ jama.2020.1585

5. WHO (2020) Clinical management of severe acute respiratory infection when Novel coronavirus ( $\mathrm{nCoV})$ infection is suspected: interim guidance. https://www.who.int/internal-publications-detai 1/clinical-managementof-severe-acute-respiratory-infection-whennovel-coronavirus-(ncov)-infection-is-suspected

6. Polosa R, Spinicci M, Prisco D (2020) "COVID-19: diagnosis, management and prognosis": a new topical collection of Internal and Emergency Medicine. Intern Emerg Med 15(5):747-750

7. Health Commission of Hunan Province. Epidemiological information of novel coronavirus pneumonia in Hunan. https://wjw. hunan.gov.cn/wjw/xxgk/gzdt/zyxw_1/202002/t20200208_11173 820.html

8. Gao HN, Lu HZ, Cao B et al (2013) Clinical findings in 111 cases of influenza A (H7N9) virus infection. N Engl J Med 368:2277-2285

9. Yang W, Lipsitch M, Shaman J (2015) Inference of seasonal and pandemic influenza transmission dynamics. Proc Natl Acad Sci USA 112:2723-2728

10. Paules C, Subbarao K (2017) Influenza. Lancet 390:697-708

11. Writing Committee of the WHO Consultation on Clinical Aspects of Pandemic (H1N1) 2009 Influenza (2010) Clinical aspects of pandemic influenza A (H1N1) virus infection. N Engl J Med 2010(362):1708-1719

12. Huang C, Wang Y, Li X, Ren L et al (2020) Clinical features of patients infected with 2019 novel coronavirus in Wuhan, China. Lancet 395(10223):497-506

13. Guan WJ, Ni ZY, Hu Y et al (2020) Clinical characteristics of coronavirus disease 2019 in China. N Engl J Med 382(18):1708-1720

14. Zhang JJ, Dong X, Cao YY et al (2020) Clinical characteristics of 140 patients infected with SARS-CoV-2 in Wuhan, China. Allergy 75(7):1730-1741

15. Chen N, Zhou M, Dong X et al (2020) Epidemiological and clinical characteristics of 99 cases of 2019 novel coronavirus pneumonia in Wuhan, China: a descriptive study. Lancet 395(10223):507-513

Publisher's Note Springer Nature remains neutral with regard to jurisdictional claims in published maps and institutional affiliations. 